\title{
Measuring motion with kinematically redundant accelerometer arrays: theory, simulation and implementation
}

Article

Accepted Version

Madgwick, S. O. H., Harrison, A. J. L., Sharkey, P. M., Vaidyanathan, R. and Harwin, W. S. (2013) Measuring motion with kinematically redundant accelerometer arrays: theory, simulation and implementation. Mechatronics, 23 (5). pp. 518529. ISSN 0957-4158 doi:

https://doi.org/10.1016/j.mechatronics.2013.04.003 Available at https://centaur.reading.ac.uk/31540/

It is advisable to refer to the publisher's version if you intend to cite from the work. See Guidance on citing.

To link to this article DOI: http://dx.doi.org/10.1016/j.mechatronics.2013.04.003

Publisher: Elsevier

Publisher statement: This is the author's version of a work that was accepted for publication in ifac-papersonline.net. Changes resulting from the publishing process, such as peer review, editing, corrections, structural formatting, and other quality control mechanisms may not be reflected in this document. Changes may have been made to this work since it was submitted for publication. A definitive version was subsequently published in ifac-papersonline.net, DOI:10.1016/j.mechatronics.2013.04.003

All outputs in CentAUR are protected by Intellectual Property Rights law, including copyright law. Copyright and IPR is retained by the creators or other 
copyright holders. Terms and conditions for use of this material are defined in the End User Agreement.

\section{www.reading.ac.uk/centaur}

\section{CentAUR}

Central Archive at the University of Reading

Reading's research outputs online 


\title{
Measuring motion with kinematically redundant accelerometer arrays: theory, simulation and implementation*
}

\author{
Sebastian O H Madgwick [s.madgwick@bris.ac.uk, +44 (0)790 333 1229] \\ Andrew J L Harrison, Paul M Sharkey,Ravi Vaidyanathan, William S Harwin
}

\begin{abstract}
This work presents two schemes of measuring the linear and angular kinematics of a rigid body using a kinematically redundant array of triple-axis accelerometers with potential applications in biomechanics. A novel angular velocity estimation algorithm is proposed and evaluated that can compensate for angular velocity errors using measurements of the direction of gravity. Analysis and discussion of optimal sensor array characteristics are provided. A damped 2 axis pendulum was used to excite all 6 DoF of the a suspended accelerometer array through determined complex motion and is the basis of both simulation and experimental studies. The relationship between accuracy and sensor redundancy is investigated for arrays of up to 100 triple axis (300 accelerometer axes) accelerometers in simulation and 10 equivalent sensors (30 accelerometer axes) in the laboratory test rig. The paper also reports on the sensor calibration techniques and hardware implementation.
\end{abstract}

\section{Introduction}

Human motion tracking for biomedical monitoring applications has received significant attention in the engineering and medical research communities in recent years (see [1, 2] for recent surveys). Stroke is one application area where Zhou [1] noted that detecting and tracking human limb movements will be essential to a home based rehabilitation service allowing the information to be used to modify movements and hence accelerate recovery.

The goal of any human motion tracking system is to collect data on human movements, and process them in a meaningful way so as to provide information pertinent to the underlying structure. To date most systems have been based on processing camera images of body worn markers [2, 3]. Body-worn sensors such as goniometers [4], pressure tubes [5], gyroscopes and accelerometers [6, 7, 8, 9, 10] have also been implemented in a range of activities and clinical conditions [10, 11, 12, 8. There are strong reasons to consider inertial-based sensors in biomechanical analysis. They are small, light-weight, low-powered, inexpensive, and unobtrusive and so can be arranged so that they do not interfere with the person's

${ }^{*}$ published in Mechatronics (Elsivier) 201323 (5). pp. 518-529 doi=10.1016/j.mechatronics.2013.04.003 
movements. They can be considered as viable for use outside a specialist laboratory and could be integrated into existing orthotics and clothing. Problems remain with pure inertial measurements since they can only provide acceleration terms and are thus sensitive to signal noise. However this work identifies two methods to reduce this problem: sensor redundancy and the constant gravity vector. Other restraints such as foot acceleration signatures or kinematic constraints can also be considered but are not covered in this paper.

Most inertial based motion tracking consists of individual units, usually consisting of a triple-axis gyroscope and a triple-axis accelerometer to estimate a state vector at a single point on the limb. This work will consider the advantages to be gained from using only tripleaxis accelerometers as the sensor[13], but recovering more accurate state information from a redundant array of such sensors. This approach is appropriate for ambulatory monitoring applications in medicine [14] and the use of redundant sensors ensures measurements are less susceptible to factors such as deformation of the soft tissues beneath an individual IMU.

The use of linear accelerometers to measure rotational kinematics has been a subject of research since the 1960s [15, 16]. For planar motion, the inverse kinematic solution is trivial [17] and has few practical applications; for example, the motion of a human knee [18. Most practical applications require that motion in all 6 Degrees Of Freedom (DoF) is accounted for. The so-called 'cube configuration' uses 6 single axis accelerometers each aligned along the diagonal of a different face of a cube. This configuration has been the focus of several studies [19, 20, 21], including an implementation in hardware [22]. Analytical [23] and empirical [24] studies have shown the 6 sensor configuration to be intrinsically unstable and limited in application of over short time intervals. Arrays of 9 accelerometers provide an alternative, stable solution [25, 26, 27].

The arrays of 6 and 9 accelerometer axis use specific configurations of multiple single-axis accelerometers. Modern MEMS accelerometers are widely available in triple-axis packages containing 3 mutually orthogonal accelerometer axes. The theoretical minimum number of triple-axis accelerometers required to measurement motion in $6 \mathrm{DoF}$ is 4 (incorporating a total of 12 linear accelerometers axis). Schemes using triple-axis accelerometers [28, 29, 30, 31, 32] do not require specific geometric specific configurations, only that the position of a sensor within an array is known. As each sensor is able to provide a vector measurement of acceleration in 3 dimensions, the physical orientation of the sensor is irrelevant provided that the orientation is known. The theoretical invariance of each sensor's position and orientation within the sensor array mean triple-axis accelerometer arrays have a clear practical advantage over single-axis accelerometer arrays.

Accelerometer arrays designed to incorporate a number of redundant sensors exploit the invariance of an inverse kinematic solution to the position of any individual sensor and such schemes typically use a matrix pseudoinverse [33, 34]. Few studies have addressed the relationship between the performance and redundancy of the system; most studies are numerical and limited to sensor arrays of no more than 24 sensitive axes [35] [34].

Due to past limitations of accelerometer technology, research into accelerometer sensors arrays has been largely limited to numerical and theoretical work. It has only been with the relatively recent developments in Microelectromechanical (MEMS) that empirical studies have became feasible [22, 28, 36, 30]. Few empirical studies exist for redundant accelerometer arrays and such studies are/have been limited to a number of sensors that represent a small margin of redundancy [37] [38]. 


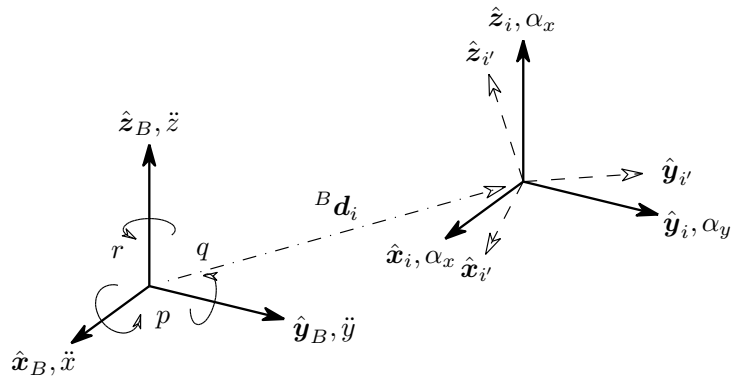

Figure 1: Schematic describing the $i$ 'th accelerometer relative to the ridged body kinematic frame

This paper presents two schemes for the measurement of translational and angular motion using a kinematically redundant array of triple-axis, accelerometers and the investigates relationship between redundancy and performance in both simulation and experimental studies. Both simulation and experimental investigations use a two axis pendulum to generate 6 DoF motion of the suspended body, enabling results to be directly compared. The redundancy of the implemented systems is significantly greater than that of previous investigations, the experimental system consists of ten triple-axis accelerometers (thirty individual accelerometer axes).

\section{Solving inverse kinematics with accelerometers}

Consider $n$ triple-axis accelerometers fixed and aligned to a rigid body such as an orthosis on a subject's limb at known positions relative to the body coordinate frame. The position of the $i$ 'th sensor is described by the vector ${ }^{B} \boldsymbol{d}_{i}$ defined in the body frame and provides the measurement vector ${ }^{i} \boldsymbol{\alpha}$. The motion of the body is described by the linear accelerations $\ddot{x}$, $\ddot{y}$ and $\ddot{z}$ of the body origin and the rotation velocities $p, q$ and $r$ around the body $x, y$ and $z$ axes respectively. This is represented as the schematic shown as Figure 1 where the mutually orthogonal unit vectors $\hat{\boldsymbol{x}}_{B}, \hat{\boldsymbol{y}}_{B}$ and $\hat{\boldsymbol{z}}_{B}$, and $\hat{\boldsymbol{x}}_{i}, \hat{\boldsymbol{y}}_{i}$ and $\hat{\boldsymbol{z}}_{i}$ define the coordinated frames of the body and $i$ 'th sensor respectively. If the $i$ 'th sensor is not aligned to the body frame then it can be considered aligned to the arbitrary frame $i^{\prime}$ defined by the mutually orthogonal unit vectors $\hat{\boldsymbol{x}}_{i^{\prime}}, \hat{\boldsymbol{y}}_{i^{\prime}}$ and $\hat{\boldsymbol{z}}_{i^{\prime}}$ (shown in Figure 1), providing the measurement vector ${ }^{i^{\prime}} \boldsymbol{\alpha}$. The orientation of frame $i^{\prime}$ relative to frame $i$ is described by the rotation matrix ${ }_{i^{\prime}}^{i} \boldsymbol{R}$ so that the aligned acceleration measurement ${ }^{i} \boldsymbol{\alpha}$ may be obtained using equation (1).

$$
{ }^{i} \boldsymbol{\alpha}={ }_{i^{\prime}}^{i} \boldsymbol{R}^{i^{\prime}} \boldsymbol{\alpha}
$$

\subsection{Forward kinematics}

The linear acceleration measured by the $i$ 'th sensor, ${ }^{i} \boldsymbol{\alpha}$, may be defined by the kinematics of the body origin using equation (2) where ${ }^{B} \boldsymbol{\omega}$ and ${ }^{B} \boldsymbol{a}$ describe the angular velocity and linear acceleration of the rigid body origin respectively. This represents the sum of linear, tangential and centripetal accelerations.

$$
{ }^{i} \boldsymbol{\alpha}={ }^{B} \dot{\boldsymbol{\omega}} \times{ }^{B} \boldsymbol{d}_{i}+{ }^{B} \boldsymbol{\omega} \times\left({ }^{B} \boldsymbol{\omega} \times{ }^{B} \boldsymbol{d}_{i}\right)+{ }^{B} \boldsymbol{a}
$$




$$
\begin{gathered}
{ }^{i} \boldsymbol{\alpha}=\left[\begin{array}{lll}
\alpha_{x} & \alpha_{y} & \alpha_{z}
\end{array}\right]^{T} \\
{ }^{B} \boldsymbol{\omega}=\left[\begin{array}{lll}
p & q & r
\end{array}\right]^{T} \\
{ }^{B} \boldsymbol{d}_{i}=\left[\begin{array}{lll}
d_{x} & d_{y} & d_{z}
\end{array}\right]^{T} \\
{ }^{B} \boldsymbol{a}=\left[\begin{array}{lll}
\ddot{x} & \ddot{y} & \ddot{z}
\end{array}\right]^{T}
\end{gathered}
$$

Equation (2) may be rearranged as equation (7) where $\boldsymbol{G}_{i}$ is a constant matrix defined by the position of the $i$ 'th sensor and $s$ is a state vector describing the kinematics of the body origin.

$$
\begin{gathered}
{ }^{i} \boldsymbol{\alpha}=\boldsymbol{G}_{i} \boldsymbol{s} \\
\boldsymbol{G}_{i}=\left[\begin{array}{cccccccccc} 
& 0 & -d_{x} & -d_{x} & 0 & d_{z} & -d_{y} & d_{y} & 0 & d_{z} \\
\boldsymbol{I}_{3} & -d_{y} & 0 & -d_{y} & -d_{z} & 0 & d_{x} & d_{x} & d_{z} & 0 \\
& -d_{z} & -d_{z} & 0 & d_{y} & -d_{x} & 0 & 0 & d_{y} & d_{x}
\end{array}\right]
\end{gathered}
$$

and

$$
\boldsymbol{s}=\left[\begin{array}{llllllllllll}
\ddot{x} & \ddot{y} & \ddot{z} & p^{2} & q^{2} & r^{2} & \dot{p} & \dot{q} & \dot{r} & p q & q r & r p
\end{array}\right]^{T}
$$

\subsection{Inverse kinematics solutions}

\subsubsection{Combinatorial method}

The inverse kinematic solution can be found directly from equation (77) through an inversion of $\boldsymbol{G}_{i}$. If the number of sensors fixed to the body is 4 then accelerometer measurements may be arranged as the vector $\boldsymbol{\alpha}_{C}$ and the corresponding square matrix $\boldsymbol{C}$ constructed as described by equations (10) and (11). $\boldsymbol{C}$ may be inverted to provide the inverse kinematic solution shown as equation (12).

$$
\begin{gathered}
\boldsymbol{\alpha}_{C}=\left[\begin{array}{llll}
{ }^{1} \boldsymbol{\alpha}^{T} & { }^{2} \boldsymbol{\alpha}^{T} & { }^{3} \boldsymbol{\alpha}^{T} & { }^{4} \boldsymbol{\alpha}^{T}
\end{array}\right]^{T} \\
\boldsymbol{C}=\left[\begin{array}{llll}
\boldsymbol{G}_{1}{ }^{T} & \boldsymbol{G}_{2}{ }^{T} & \boldsymbol{G}_{3}{ }^{T} & \boldsymbol{G}_{4}{ }^{T}
\end{array}\right]^{T} \\
\boldsymbol{s}=\boldsymbol{C}^{-1} \boldsymbol{\alpha}_{C}
\end{gathered}
$$

This solution cannot directly incorporate $n$ triple-axis accelerometer for $n \neq 4$. If $n>4$ then there exists $m$ unique combinations of sensors and possible $\boldsymbol{C}$ matrices where $m$ is defined by equation (13). A single value of $s$ may be computed as the mean of the $m$ possible values, as described by equation (14) where $\boldsymbol{s}_{j}$ a value for a given combination of four triple-axis accelerometer.

$$
\begin{gathered}
m=\left(\begin{array}{l}
n \\
4
\end{array}\right)=\frac{n !}{24(n-4) !} \\
\overline{\boldsymbol{s}}=\frac{1}{m} \sum_{j=0}^{m} \boldsymbol{s}_{j}
\end{gathered}
$$

If the positions of any sensors are equal or if all sensors exist on a plane (for a non-planar system) then $\boldsymbol{G}_{i}$ is singular and cannot be inverted. For $n>4$, a unique value of $\boldsymbol{C}^{-1}$ must be computed for each combination. This method may result in a considerable computational load as an array of $n$ sensors requires $m 12 \times 12$ matrix inversions and multiplications. 


\subsubsection{Pseudoinverse method}

For $n$ accelerometer measurements arranged in the vector $\boldsymbol{\alpha}_{P}$, the non-square matrix $\boldsymbol{P}$ may be constructed and the inverse kinematic solution may be computed as equation (17) where + denotes the pseudoinverse. This is a similar approach to that of existing inverse kinematic solutions for redundant accelerometer arrays [33, 34].

$$
\begin{gathered}
\boldsymbol{\alpha}_{P}=\left[\begin{array}{lllll}
{ }^{1} \boldsymbol{\alpha}^{T} & { }^{2} \boldsymbol{\alpha}^{T} & { }^{3} \boldsymbol{\alpha}^{T} & \ldots & { }^{n} \boldsymbol{\alpha}^{T}
\end{array}\right]^{T} \\
\boldsymbol{P}=\left[\begin{array}{lllll}
\boldsymbol{G}_{1}{ }^{T} & \boldsymbol{G}_{2}{ }^{T} & \boldsymbol{G}_{3}{ }^{T} & \ldots & \boldsymbol{G}_{n}{ }^{T}
\end{array}\right]^{T} \\
\boldsymbol{s}=\boldsymbol{P}^{+} \boldsymbol{\alpha}_{P}
\end{gathered}
$$

The Moore-Penrose pseudoinverse of $\boldsymbol{P}$ may be calculated as equation (18) provided that the rows or $\boldsymbol{P}$ are linearly independent and the matrix represents $>4$ sensors.

$$
\boldsymbol{P}^{+}=\left(\boldsymbol{P}^{T} \boldsymbol{P}\right)^{-1} \boldsymbol{P}^{T}
$$

If the positions of any sensors are equal or if all sensors exist on a plane (for a non-planar system) then the rows of $\boldsymbol{P}$ are not linearly independent and equation (18) may not be used. The computational requirement of this method considerably is less than that of the combinatorial method as only one $n \times 12$ matrix inversion and multiplication is required for $n$ sensors.

It should also be noted that the pseudoinverse is identical to an inverse computed using the non-zero singular values from a singular value decomposition of the matrix. Thus the sensitivity of the solution can be evaluated from the ratio of the largest to the smallest singular value (equivalent to the condition number of the matrix) as well as the composition of the two uniary matrices associated with the decomposition. This analysis confirms the findings presented later in ??, optimal array configurations. Other matrix methods are available to solve equation (17) such as QR decomposition with back substitution, or minimising the $l_{1}$ norm.

\subsection{Angular velocity estimation}

The state vector $\boldsymbol{s}$ does not directly yield individual angular velocity terms. This is a problem common to all accelerometer-only inertial measurement methods and many solutions have proposed. Cardou et al. provide a discussion and analysis of the different approaches to this problem and present a method more robust than many existing solutions [39].

Here we propose a novel angular velocity estimation algorithm that employs the gradient descent approach but combines this with a complementary filter structure to achieve advantages in computational efficiency and well described error dynamics. The method is similar to that described in Madgwick et al [40]. The angular velocity estimation is further improved by the orientation estimation algorithm presented in section 3 to compensate for bias errors in the estimated angular velocity.

The available angular velocity terms may be used to construct an objective function $\boldsymbol{f}(\tilde{\boldsymbol{\omega}}, \boldsymbol{s})$ as shown in equation (19) where $\tilde{\boldsymbol{\omega}}$ is the estimated angular velocity. If $\boldsymbol{f}(\tilde{\boldsymbol{\omega}}, \boldsymbol{s})=0$ 
then $\tilde{\boldsymbol{\omega}}= \pm \boldsymbol{\omega}$. As each angular velocity term is only available as the product of itself or another, the sign of each quantity is lost and cannot be recovered directly.

$$
\begin{gathered}
\boldsymbol{f}(\tilde{\boldsymbol{\omega}}, \boldsymbol{s})=\left[\begin{array}{c}
\tilde{p}^{2}-p^{2} \\
\tilde{q}^{2}-q^{2} \\
\tilde{r}^{2}-r^{2} \\
\tilde{p} \tilde{q}-p q \\
\tilde{q} \tilde{r}-q r \\
\tilde{r} \tilde{p}-r p
\end{array}\right] \\
\tilde{\boldsymbol{\omega}}=\left[\begin{array}{lll}
\tilde{p} & \tilde{q} & \tilde{r}
\end{array}\right]^{T}
\end{gathered}
$$

The gradient of the solution surface (defined by $\eta_{p}, \eta_{q}$ and $\eta_{r}$ ) created by $\boldsymbol{f}(\tilde{\boldsymbol{\omega}}, \boldsymbol{s})$ may be used to compute the direction of the error in the estimated angular velocity as equation (23) where $\boldsymbol{J}_{\boldsymbol{f}}$ is the Jacobian of $\boldsymbol{f}(\tilde{\boldsymbol{\omega}}, \boldsymbol{s})$.

$$
\begin{aligned}
{\left[\begin{array}{l}
\eta_{p} \\
\eta_{q} \\
\eta_{r}
\end{array}\right]=} & \boldsymbol{J}_{\boldsymbol{f}}^{T}(\tilde{\boldsymbol{\omega}}) \boldsymbol{f}(\tilde{\boldsymbol{\omega}}, \boldsymbol{s}) \\
= & {\left[\begin{array}{cccccc}
2 \tilde{p} & 0 & 0 & \tilde{q} & 0 & \tilde{r} \\
0 & 2 \tilde{q} & 0 & \tilde{p} & \tilde{r} & 0 \\
0 & 0 & 2 \tilde{r} & 0 & \tilde{q} & \tilde{p}
\end{array}\right]^{T}\left[\begin{array}{l}
\tilde{p}^{2}-p^{2} \\
\tilde{q}^{2}-q^{2} \\
\tilde{r}^{2}-r^{2} \\
\tilde{p} \tilde{q}-p q \\
\tilde{q} \tilde{r}-q r \\
\tilde{r} \tilde{p}-r p
\end{array}\right] } \\
= & {\left[\begin{array}{ll}
2 \tilde{p}\left(\tilde{p}^{2}-p^{2}\right)+\tilde{q}(\tilde{p} \tilde{q}-p q)+\tilde{r}(\tilde{r} \tilde{p}-r p) \\
2 \tilde{q}\left(\tilde{q}^{2}-q^{2}\right)+\tilde{p}(\tilde{p} \tilde{q}-p q)+\tilde{r}(\tilde{q} \tilde{r}-q r) \\
2 \tilde{r}\left(\tilde{r}^{2}-r^{2}\right)+\tilde{q}(\tilde{q} \tilde{r}-q r)+\tilde{p}(\tilde{r} \tilde{p}-r p)
\end{array}\right] }
\end{aligned}
$$

The direction of this vector may be combined with a magnitude computed as the difference between the magnitudes of the estimated angular velocities and actual angular velocities. The estimated error in the angular velocity, $\tilde{\boldsymbol{e}}$, is therefore calculated as the dot product shown in equation (24). Due to the loss of sign in the available angular velocity terms the error will be computed relative to the closest value of $\pm \boldsymbol{\omega}$.

$$
\tilde{\boldsymbol{e}}=\left[\begin{array}{c}
\operatorname{sign}\left(\eta_{p}\right) \\
\operatorname{sign}\left(\eta_{q}\right) \\
\operatorname{sign}\left(\eta_{r}\right)
\end{array}\right] \cdot\left[\begin{array}{c}
|| \tilde{p} \mid-\sqrt{p^{2}} \\
|\tilde{q}|-\sqrt{q^{2}} \mid \\
|\tilde{r}|-\sqrt{r^{2}} \mid
\end{array}\right]
$$

The estimated angular velocity is computed from the angular acceleration and estimated error in the angular velocity using equation (25). This is represented as the block diagram shown as Figure 2,

$$
\tilde{\boldsymbol{\omega}}=\int(\dot{\boldsymbol{\omega}}-K \tilde{\boldsymbol{e}})
$$




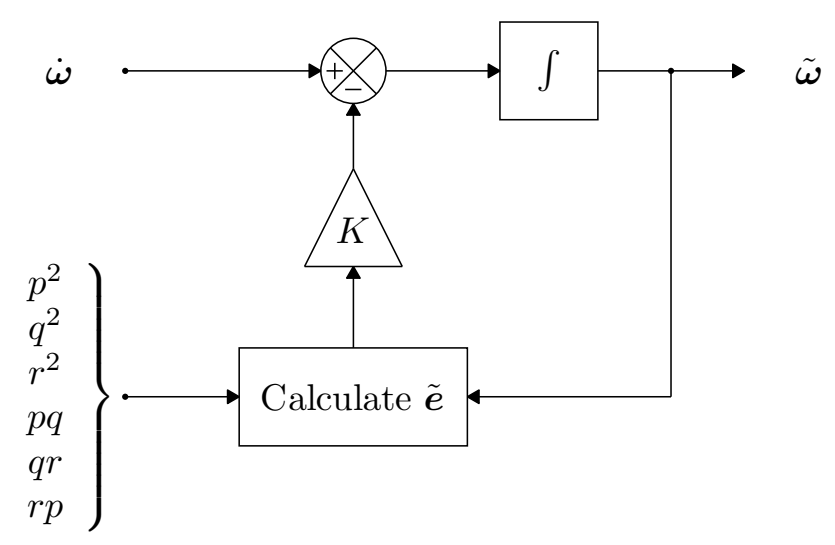

Figure 2: Complementary filter used for angular velocity estimation

\subsubsection{Stability and response to initial conditions}

A simplified analysis of the proposed complementary filter is possible if the estimated error term, $\tilde{\boldsymbol{e}}$, is assumed to be equal to the true error term, $\boldsymbol{e}$. The complementary filter can then be seen to represent a first order system with error dynamics governed by equation (26). The system is therefore stable for all values of $K>0$ and the error can be expected to decay exponentially at a rate governed by $K$.

$$
\dot{e}=-K e
$$

As the estimated error may only be computed relative to the closest value of $\pm \boldsymbol{\omega}, \tilde{\boldsymbol{\omega}}$ will converge to the closest value of either $\pm \boldsymbol{\omega}$. A correct sign assertion may be assured if the dynamics in $\dot{\boldsymbol{\omega}}$ are of a sufficiently large magnitude relative to the convergence rate of $\tilde{\boldsymbol{\omega}}$ governed by $K$, at the zero-crossing point of $\boldsymbol{\omega}$. A sufficient magnitude of $\dot{\boldsymbol{\omega}}$ relative to the convergence rate of $\tilde{\boldsymbol{\omega}}$ would mean that integral drift would not dominate the $\tilde{\boldsymbol{\omega}}$ dynamics and the value of $\tilde{\boldsymbol{\omega}}$ would not drift across zero and hence cause an incorrect sign.

\section{Orientation estimation and angular velocity compen- sation}

In many applications it can be assumed that the mean linear accelerations measured over an extended period of time represents gravity. The direction of gravity measured relative to the acceleormeter array may therefore be used to estimate the attitude of the array relative to the Earth's surface. As will be shown in section 6.3, sensor measurement and alignment errors result in a bias error in the estimated angular velocity at low velocities. Knowledge of the accelerometer array orientation may be used to correct for steady state errors in the estimation angular velocity.

Mahony et al. [41] have presented an orientation estimation algorithm for conventional IMUs consisting of gyroscopes and accelerometers. The algorithm is equally applicable to the accelerometer array presented in this paper as the inverse kinematic solution yields linear accelerations and angular velocities. Equations (27) to (30) represent a quaternion 
implementation [42] of this algorithm where ${ }_{E}^{B} \hat{\boldsymbol{q}}$ is the estimated orientation of the Earth relative to the body, represented as a quaternion of elements $q_{0}$ to $q_{3}$. The gains $K_{P}$ and $K_{I}$ represent proportional and integral gains respectively. The ^accent denotes the normalised unit vector and the $\otimes$ operator denotes a quaternion product.

$$
\begin{gathered}
{ }_{E}^{B} \boldsymbol{q}=\int{ }_{E}^{B} \dot{\boldsymbol{q}} \\
{ }_{E}^{B} \dot{\boldsymbol{q}}={ }_{2}^{1}{ }_{E}^{B} \hat{\boldsymbol{q}} \otimes\left[0 \quad\left({ }^{B} \boldsymbol{\omega}-\boldsymbol{\delta}\right)^{T}\right] \\
\boldsymbol{\delta}=K_{P} \boldsymbol{\epsilon}+K_{I} \int \boldsymbol{\epsilon} \\
\boldsymbol{\epsilon}={ }^{B} \boldsymbol{a} \times\left[\begin{array}{c}
2 q_{1} q_{3}-q_{0} q_{2} \\
2 q_{0} q_{1}+q_{2} q_{3} \\
q_{0}^{2}-q_{1}^{2}-q_{2}^{2}+q_{3}^{2}
\end{array}\right]
\end{gathered}
$$

The error in the estimated angular velocity is represented by the integral feedback term. The compensated estimated angular velocity is therefore obtained as equation (31).

$$
\tilde{\boldsymbol{\omega}}^{\prime}=\tilde{\boldsymbol{\omega}}-K_{i} \int \boldsymbol{\epsilon}
$$

\section{2 axis pendulum kinematics}

A 2 axis pendulum was the chosen subject of simulation and experimental studies as it approximates a number of human joints and the oscillatory motion is analogous to gait and excites all 6 DoF of the suspended body. The damped motion results in both low and high magnitude rates and the complex motion of the 2 axis joint results in rotational kinematics in all three axes of the suspended body. The pendulum is represented by the schematic shown as Figure 3 where $\theta_{1}$ and $\theta_{2}$ represent the angle in each axis of the joint. The 2 axis pendulum equations of motion have been derived as equations (32) and (33) where $c_{1}$ and $c_{2}$ are the damping ratio of each axis of the joint.

$$
\begin{gathered}
\ddot{\theta}_{1}=2 \dot{\theta}_{1} \dot{\theta}_{2} \tan \theta_{2}-\frac{g \sin \theta_{1}}{l \cos \theta_{2}}-\frac{c_{1} \dot{\theta}_{1}}{m \cos ^{2} \theta_{2}} \\
\ddot{\theta}_{2}=-\dot{\theta}_{1}^{2} \cos \theta_{2} \sin \theta_{2}-\frac{g}{l} \cos \theta_{1} \sin \theta_{2}-\frac{c_{2} \dot{\theta}_{2}}{m}
\end{gathered}
$$

The body kinematics are computed as the forward kinematics described by equations (34) to (37) where ${ }^{B} \boldsymbol{a},{ }^{B} \boldsymbol{\omega}$ and ${ }^{B} \dot{\boldsymbol{\omega}}$ describe the translational acceleration, angular velocity and angular acceleration respectively of the body frame in directions relative to the body frame. ${ }_{B}^{E} \boldsymbol{R}$ describes the orientation of the body frame relative to the Earth frame.

$$
{ }^{B} \boldsymbol{a}=g\left[\begin{array}{c}
-\sin \theta_{2} \\
\sin \theta_{1} \cos \theta_{2} \\
\cos \theta_{1} \cos \theta_{2}
\end{array}\right]
$$




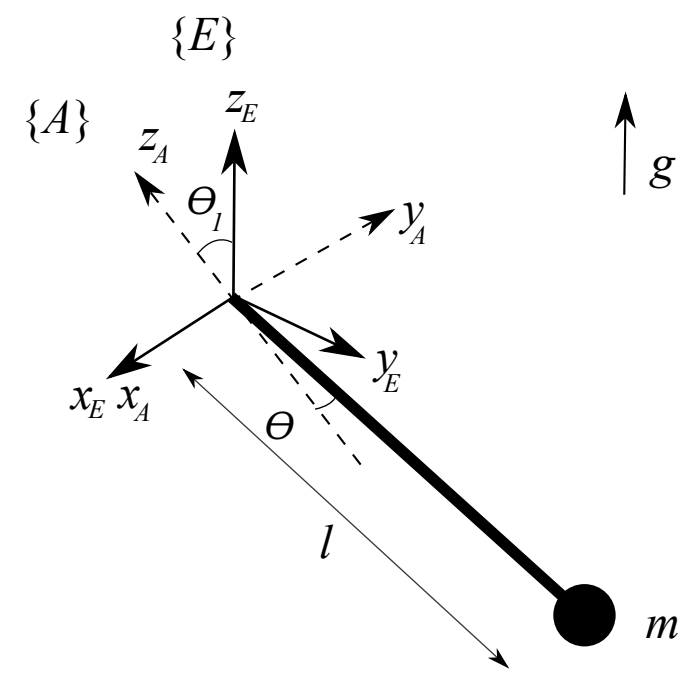

Figure 3: 2 axis pendulum schematic

$$
\begin{gathered}
{ }^{B} \boldsymbol{\omega}=\left[\begin{array}{c}
\dot{\theta}_{1} \cos \theta_{2} \\
\dot{\theta}_{2} \\
\dot{\theta}_{1} \sin \theta_{2}
\end{array}\right] \\
{ }^{B} \dot{\boldsymbol{\omega}}=\left[\begin{array}{cc}
\ddot{\theta}_{1} \cos \theta_{2}-\dot{\theta}_{1} \dot{\theta}_{2} \sin \theta_{2} \\
\ddot{\theta}_{2} \\
\ddot{\theta}_{1} \sin \theta_{2}+\dot{\theta}_{1} \dot{\theta}_{2} \cos \theta_{2}
\end{array}\right] \\
{ }_{B}^{E} \boldsymbol{R}=\left[\begin{array}{ccc}
\cos \theta_{2} & 0 & \sin \theta_{2} \\
\sin \theta_{1} \sin \theta_{2} & \cos \theta_{1} & -\sin \theta_{1} \cos \theta_{2} \\
-\cos \theta_{1} \sin \theta_{2} & \sin \theta_{1} & \cos \theta_{1} \cos \theta_{2}
\end{array}\right]
\end{gathered}
$$

\section{$5 \quad$ Simulation studies}

Simulation studies were used for the initial testing of the proposed methods and to investigate the relationship between system accuracy and redundancy. Simulations used the damped 2 axis pendulum model with an array of $n$ triple-axis accelerometers positioned evenly around a $360^{\circ}$ helix concentric with the length of the pendulum and with a radius of $0.05 \mathrm{~m}$. Figure 4 shows the pendulum and triple-axis accelerometers as drawn by the simulation software. For convenience, all sensors were aligned with the pendulum body coordinate frame; arbitrary orientations are used and calibrated for in the experimental studies discussed in section 6

The forward kinematics were computed to provide ideal sensor accelerometer measurements and processed through a sensor model to simulate the frequency response and noise characteristics. The sensor model was a simplification of a Simulink based accelerometer behavioural model provided by the manufacturer (Analog Devices). The noise model consisted of zero mean additive Gaussian noise with a standard deviation of $0.045 \mathrm{~ms}^{-2}$ chosen to match the experimental data. The signal was then processed through a first order low-pass filter with a cut-off frequency of $50 \mathrm{~Hz}$. The angular velocity estimation filter used a fixed gain of $K=0.5$. Pendulum model parameters were fixed so that $m=0.5 \mathrm{~kg}, l=0.3 \mathrm{~m}$, 


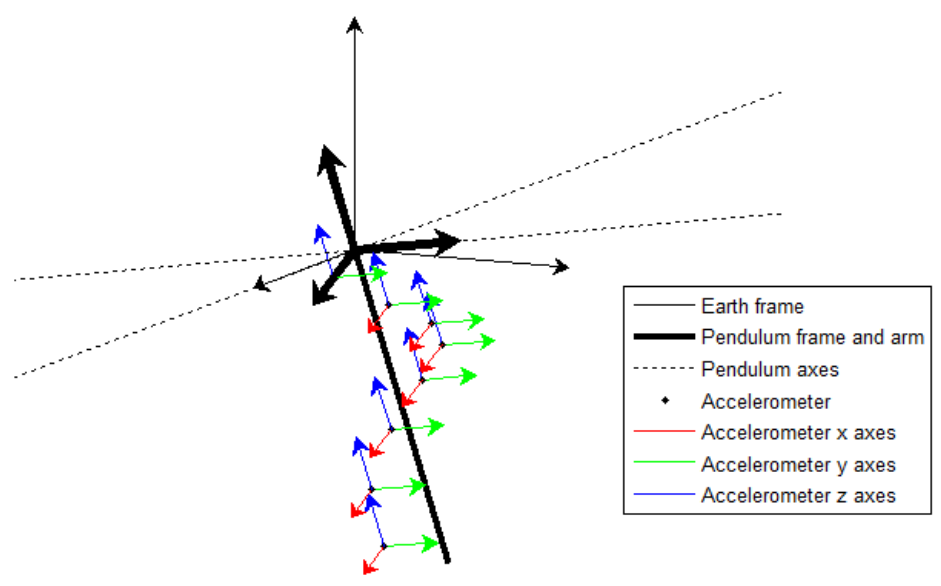

Figure 4: Schematic of pendulum with triple-axis accelerometers mounted in helix pattern

$g=9.8 \mathrm{~ms}^{-2}, c_{1}=0.1 \mathrm{Nsm}^{-1}$ and $c_{2}=0.3 \mathrm{Nsm}^{-1}$, with initial conditions of $\theta_{1}=\theta_{2}=60^{\circ}$, and $\dot{\theta}_{1}=\dot{\theta}_{2}=0$. The simulation was run for 30 seconds with a discrete-time step of $1 \mathrm{~ms}$.

To investigate the relationship between the redundancy and accuracy of a sensor array, simulations were conducted for arrays consisting of between 5 and 100 triple-axis sensors. For each array the mean of the RMS errors in the $x, y$ and $z$ dimensions was computed for the linear acceleration, angular acceleration and angular velocity. The error in the estimated orientation was not investigated as integral drift would fail to represent a meaningful relationship. Results were obtained using both the combinatorial and pseudoinverse method to enable a comparison.

The integral drift inherent to the angular velocity estimation algorithm would introduce a bias in the estimated velocity at low rates. This random steady state error meant that the apparent relationship between the angular velocity error and redundancy was non-monotonic. To compensate for this, the mean result of 5 simulations was computed for each value of redundancy. The results for angular acceleration and velocity are shown as Figure 5 and Figure 6. It can be seen that the combinatorial method results in an greater error than the pseudoinverse method at greater computational expense. Simulations of the combinatorial method for arrays $>33$ sensors were abandoned as the time taken to process arrays of this size became impractical. The non-monotonic relationship observed in the estimated angular velocity is due to the 'random walk' in this state that comes about at low velocities and is constrained by the feedback in the angular velocity estimation filter. This random walk is due to the noise (in simulation) of the Analog Devices accelerometers. As the angular velocity is reduced (due to damping), noise dominates the signal and the estimated angular velocity is then largely governed by the integration of the angular acceleration which again is largely made up of the sensor noise causing a random walk. This error does not directly relate to the number of sensors.

Oscillatory motion of the pendulum and the frequency response of the sensors results in a minimum achievable error represented by the oscillatory error components due to the phaselag of the sensor outputs (due to a simulated anti-aliasing filter). This minimum achievable error was computed by running a simulation using an accelerometer error model comprised 


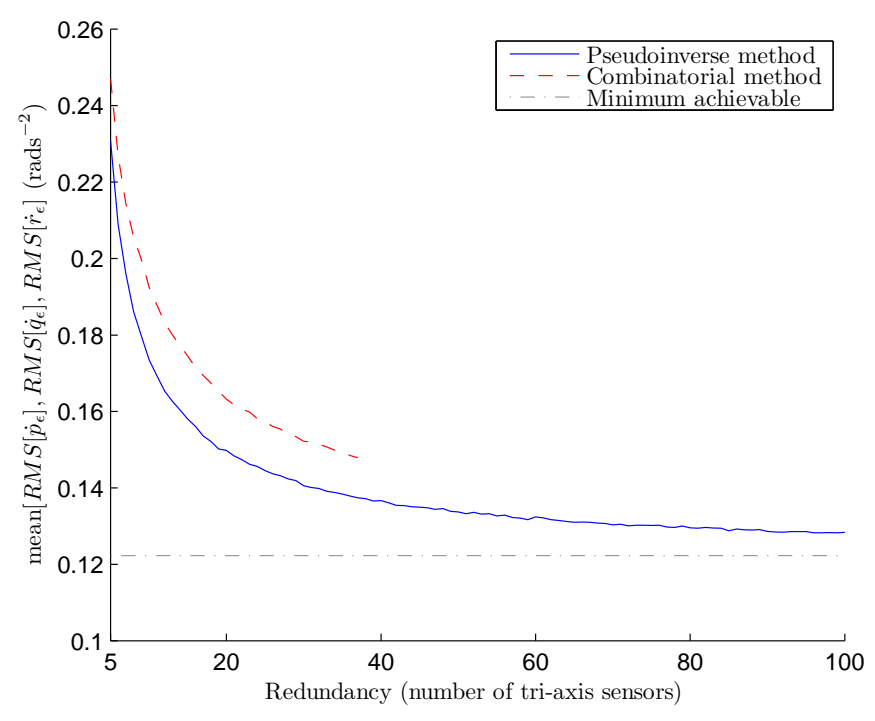

Figure 5: Angular acceleration error vs. sensor redundancy

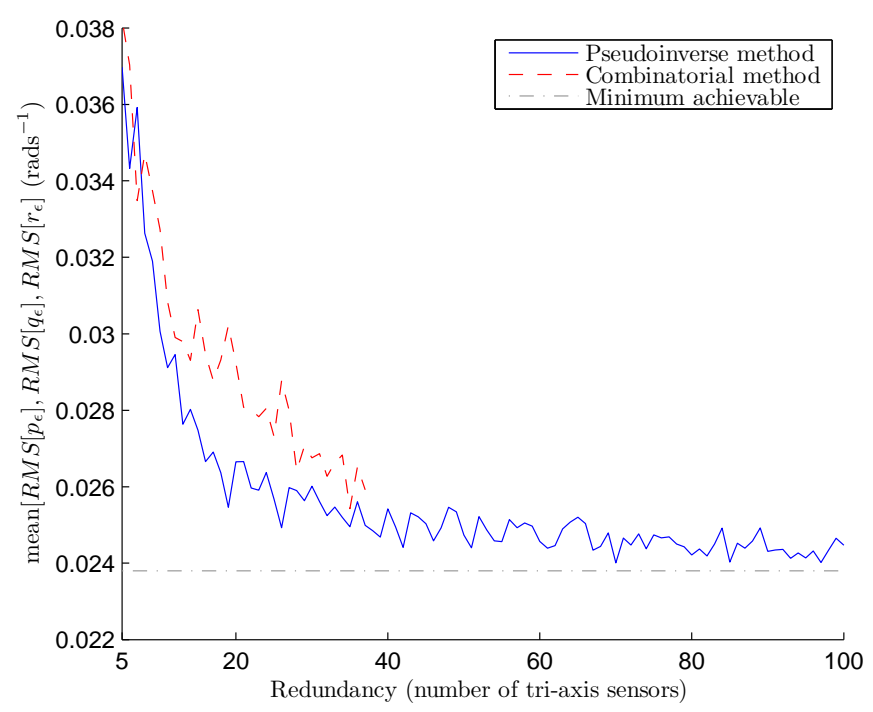

Figure 6: Angular velocity error vs. sensor redundancy

of only the anti-aliasing filter, i.e. zero noise. The resultant error is the same for arrays of all sizes and represents the minimum achieve error. The respective minimum achieve errors are indicated in Figure 5 and Figure 6.

The pendulum testbed allows more complex motion to be evaluated. If the initial conditions use a nonzero velocity vector and dissimilar damping coefficients, all cross terms in equation 7 can be elicited at a range of frequencies. The spectrum of any individual accelerometer axis will contains frequencies up to the natural frequency of the pendulum and hence the results can be demonstrated to work across this frequency range. The upper graphs in figure 6 and 7 demonstrate this result for a simulation of a cluster of 20 accelerometers placed in a $5 \mathrm{~cm}$ radius helix beginning half way down the pendulum and continuing to the 

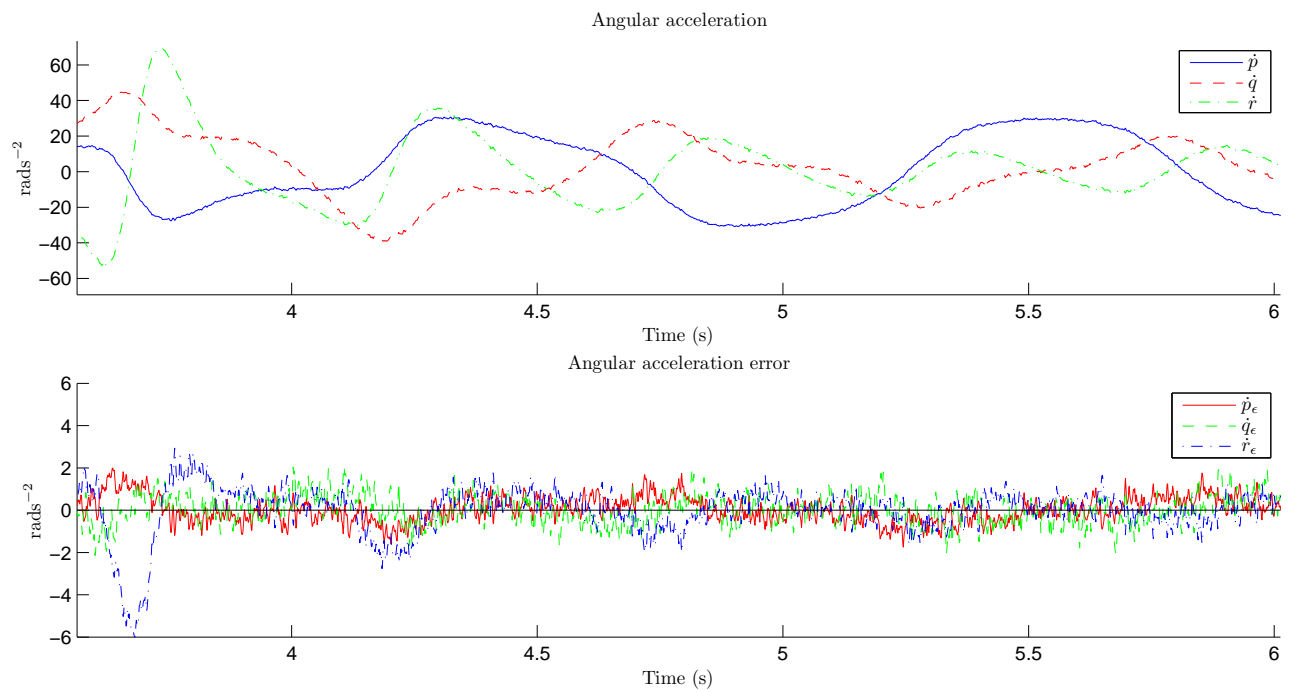

Figure 7: Angular accelerations of pendulum measured by accelerometer array (top) and residuals (bottom)
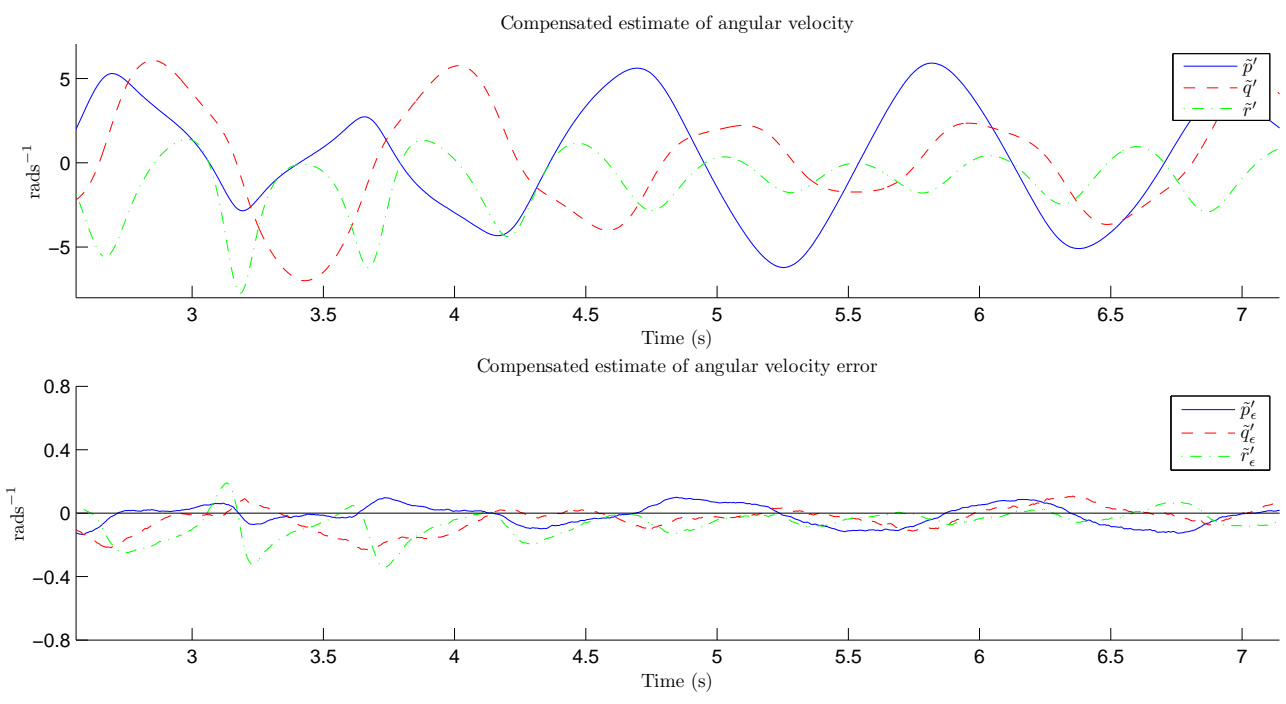

Figure 8: Estimated angular velocity compensated by orientation estimation algorithm (top) and residuals (bottom)

end. When viewed in isolation this is readily observed to be a non obvious complex motion. (If acceptable to the editors, a footnote could be included here with the URL of a YouTube video). The error residuals are given in the lower graphs of Figures 7 and 8 , 


\section{$6 \quad$ Experimental studies}

The experimental studies were conducted to validate the above simulation results. The studies were designed to be as similar to the simulation studies as possible and used a redundant array of 10 triple-axis accelerometers. Unlike the simulation studies, the experimental studies were subject to 2 significant sources of error: sensor calibration errors (of gains and biases) and sensor installation errors (of position and alignment).

\subsection{Hardware}

Experimental studies required data to be acquired from an array of 10 triple-axis accelerometers at a sample rate of at least $1 \mathrm{kHz}$ and with as high a resolution as practicable; sensor resolution had been identified as a limiting factor in similar studies [17]. To meet these requirements, custom equipment was developed for this research project. The equipment consisted of the DAQ32 data acquisition board, the DAQ32 PC Interface software and 10 triple-axis accelerometer probes.

\subsubsection{Data acquisition equipment}

The DAQ32 is a 32 channel USB data acquisition board built around Microchip's highend dsPIC33FJ256GP710 Digital Signal Controller. The DAQ32 features a filtered power supply for analogue sensors and 32 analogue input channels each sampled at $1.024 \mathrm{kHz}$. Each channel is oversampled by a factor of 8 and the truncated mean result used for enhanced filtering and accuracy.

\subsubsection{Accelerometer probes}

Each accelerometer probe consisted of an Analog Devices' ADXL335 triple-axis accelerometer with signal and power supply conditioning circuitry assembled on the accelerometer package to minimise size and weight. The probe's $0.4 \mathrm{~m}$ lead consisted of wires twisted together so that the power supply wires would provide a level of electromagnetic shielding to the accelerometer signals. Fine wire connections were sunk in a silicon sealant to provide mechanical durability. The complete probe (shown in Figure 9) occupied a volume of a 5 mm cube and weighed less than $1 \mathrm{~g}$ (excluding lead).

\subsubsection{Test rig}

The pendulum was constructed from 2 perpendicular sheets of aluminium so that the 10 accelerometer probes on the surface of the sheets formed an approximate helix with a length of $350 \mathrm{~mm}$ and radius of $50 \mathrm{~mm}$. Each accelerometer was mounted at arbitrary orientations varying up to $180^{\circ}$. The position of each accelerometer was measured. The pendulum joint was constructed from 2 precision servo potentiometers so that angular position of each axis could be measured. The 32 analogue channels required by the 10 triple-axis accelerometer probes and 2 potentiometers were all accommodated by the DAQ32. Figure 10 shows the pendulum with attached accelerometer probes and annotated potentiometer axes $\theta_{1}$ and $\theta_{2}$. 

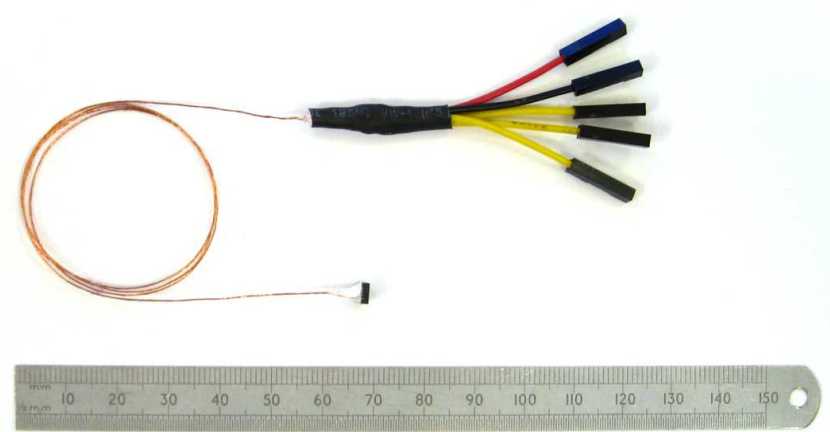

Figure 9: Complete accelerometer probe next to a $150 \mathrm{~mm}$ ruler

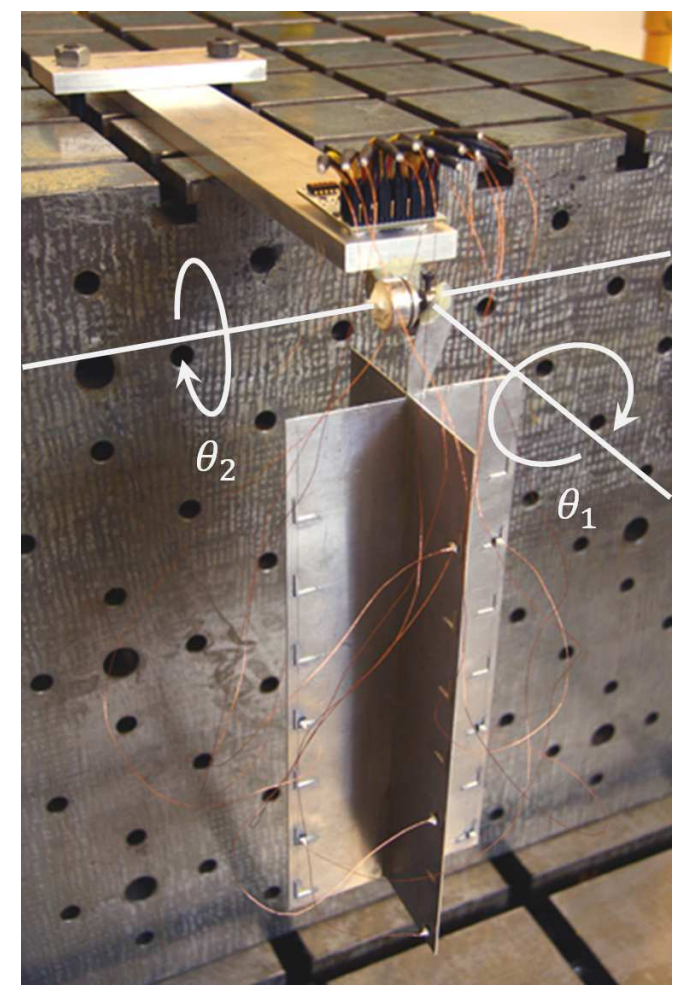

Figure 10: 2 axis pendulum with attached accelerometer probes and annotated potentiometer axes $\theta_{1}$ and $\theta_{2}$

Simulation was based on a helix, the experimental rig needed to be as rigid as possible so a cross structure was chosen. This is equivalent to a four-spoke helix. This is a reasonable compromise to allow the experimental rig to be manufactured. The cross structure in the pendulum allows for greater rigidity in the pendulum and higher accuracy when placing the sensors. 


\subsection{Calibration}

\subsubsection{Axis gain and bias}

As the accelerometer output is linear, the calibrated measurement, $\boldsymbol{\alpha}$, may be obtained from the un-calibrated measurement, $\boldsymbol{u}$, using the relationship described by (38). The vector $\boldsymbol{b}$ and diagonal matrix $\boldsymbol{K}$ account for the individual axis bias and sensitivity respectively. The skew matrix $\boldsymbol{S}$ accounts for non-orthogonality and coupling between the accelerometer axes. The gain $s_{m n}$ defines the proportion of axis $n$ affecting axis $m$.

$$
\begin{aligned}
\boldsymbol{\alpha} & =\boldsymbol{S} \boldsymbol{K} \boldsymbol{u}-\boldsymbol{b} \\
& =\left[\begin{array}{ccc}
1 & s_{x y} & s_{x z} \\
s_{y x} & 1 & s_{y z} \\
s_{z x} & s_{z y} & 1
\end{array}\right]\left[\begin{array}{ccc}
k_{x} & 0 & 0 \\
0 & k_{y} & 0 \\
0 & 0 & k_{z}
\end{array}\right]\left[\begin{array}{l}
u_{x} \\
u_{y} \\
u_{z}
\end{array}\right]-\left[\begin{array}{l}
b_{x} \\
b_{y} \\
b_{z}
\end{array}\right]
\end{aligned}
$$

A stationary accelerometer will measure the direction and magnitude of the Earth's gravitational field due to the reaction force on the mass within the MEMS structure. For a calibrated sensor, the measured magnitude will be constant at all orientations of the sensor. That is, equation (39) will be true for all said measurements where $g$ is the magnitude of gravity. Therefore, $\boldsymbol{K}$ and $\boldsymbol{b}$ may be found as the solution to equation 40 where $\boldsymbol{u}_{j}$ represents the un-calibrated sensor measurement at the $j$ 'th orientation within a calibration dataset. Although it is possible to compound $\boldsymbol{S}$ and $\boldsymbol{K}$ in to a single matrix, it was found this would limit the success of an optimisation algorithm as it became sensitive to the initial guesses of the gain parameters.

$$
\begin{gathered}
g=\|\boldsymbol{S} \boldsymbol{K} \boldsymbol{u}-\boldsymbol{b}\| \\
\min _{\boldsymbol{S}, \boldsymbol{K}, \boldsymbol{b} \in \Re} \sum_{j}\left(g-\left\|\boldsymbol{S} \boldsymbol{K} \boldsymbol{u}_{j}-\boldsymbol{b}\right\|\right)^{2}
\end{gathered}
$$

A calibration dataset was collected using an automated calibration gimbal capable of rotating independently around the pan, roll and tilt axes. The pendulum (with attached accelerometer probes) was fixed to the gimbal so that it could be rotated to any orientation. An automated routine was composed to rotate the gimbal pitch and roll axis through their full range in $10^{\circ}$ steps so that every combination of pitch and roll angle was achieved. At each orientation, the gimbal was allowed 10 seconds to come to rest and then the mean of each accelerometer output was taken for a 15 second period.

A value of $g$ was obtained as $9.812 \mathrm{~ms}^{-2}$ for the geographical location of the studies [43]. Equation (40) was solved using MATLAB's Optimisation toolbox's fminunc function and the Broyden-Fletcher-Goldfarb-Shanno (BFGS) method. Initial values for gains and biases were chosen as the typical values detailed in the AXDL335 datasheet. Calibration was verified by comparing the measured magnitude of gravity with the known magnitude at each orientation within the calibration dataset. Figure 11] shows typical calibrated measurements of one accelerometer for the full calibration dataset.

\subsubsection{Alignment}

Hardware installation errors mean that a misalignment must be accounted for in each tripleaxis accelerometer. Alignment errors as low as $0.1^{\circ}$ have been shown to result in significant 


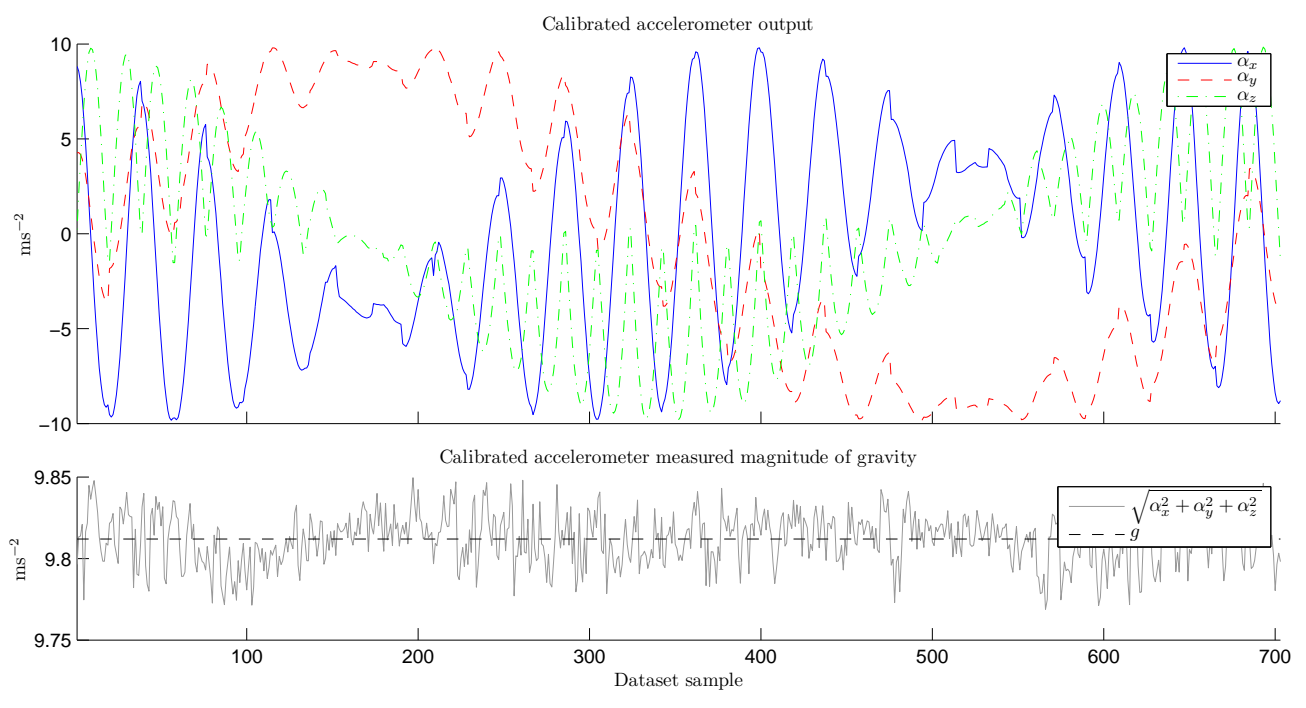

Figure 11: Validation the calibrated accelerometer gains and biases

errors in estimated kinematic states $[33$. An aligned triple-axis accelerometer measurement, ${ }^{i} \boldsymbol{\alpha}$, is related to the misaligned measurement ${ }^{i^{\prime}} \boldsymbol{\alpha}$ by the rotation matrix ${ }_{i^{\prime}}^{i} \boldsymbol{R}$ as described by equation (11). The alignment may be equivalently represented by the quaternion ${ }_{i^{\prime}}^{i} \boldsymbol{q}$. If the array is stationary, equation (41) will be true for sensors at all orientations. Therefore ${ }_{i^{\prime}}^{i} \boldsymbol{q}$ may be found as the solution to (42) where ${ }^{i^{\prime}} \boldsymbol{\alpha}_{j}$ and ${ }^{B} \boldsymbol{a}_{j}$ represent the measured acceleration of the $i$ 'th accelerometer and the acceleration of the body at the $j$ 'th orientation respectively. As the acceleration of the body is unknown, one fiducial sensor must be chosen to provide a measurement equal to ${ }^{B} \boldsymbol{a}$. An alternative kinematic minimisation approach for alignment calibration was also implemented but performance was found to be no better than simply choosing a convenient sensor to be the fiducial.

$$
\begin{gathered}
{ }^{B} \boldsymbol{a}={ }_{i^{\prime}}^{i} \hat{\boldsymbol{q}}^{*} \otimes{ }^{i^{\prime}} \boldsymbol{\alpha} \otimes{ }_{i^{\prime}}^{i} \hat{\boldsymbol{q}} \\
\min _{\substack{i \\
i^{\prime}}} \sum_{j}\left[{ }_{i^{\prime}}^{i} \hat{\boldsymbol{q}}^{*} \otimes{ }^{i^{\prime}} \boldsymbol{\alpha}_{j} \otimes{ }_{i^{\prime}}^{i} \hat{\boldsymbol{q}}-{ }^{B} \boldsymbol{a}_{j}\right]^{2}
\end{gathered}
$$

Equation (42) was solved using the same calibration dataset and method described in section 6.2.1. The fiducial sensor was chosen as that judged to be best aligned to the pendulum on visual inspection. This gravitational based attitude calibration scheme is similar to previously proposed techniques; however, the use of a quaternion representation avoids problems of non-orthogonality associated with a rotation matrix based approach [44].

\subsection{Results}

The pendulum was left to swing with motion in both axis remain in phase until rest while data from each of the 10 triple-axis accelerometers and 2 joint potentiometers was recorded. The 'true' suspended body kinematics were then estimated for the first 30 seconds of the dataset using the potentiometer data and kinematic model within a moving horizon state estimation algorithm. 

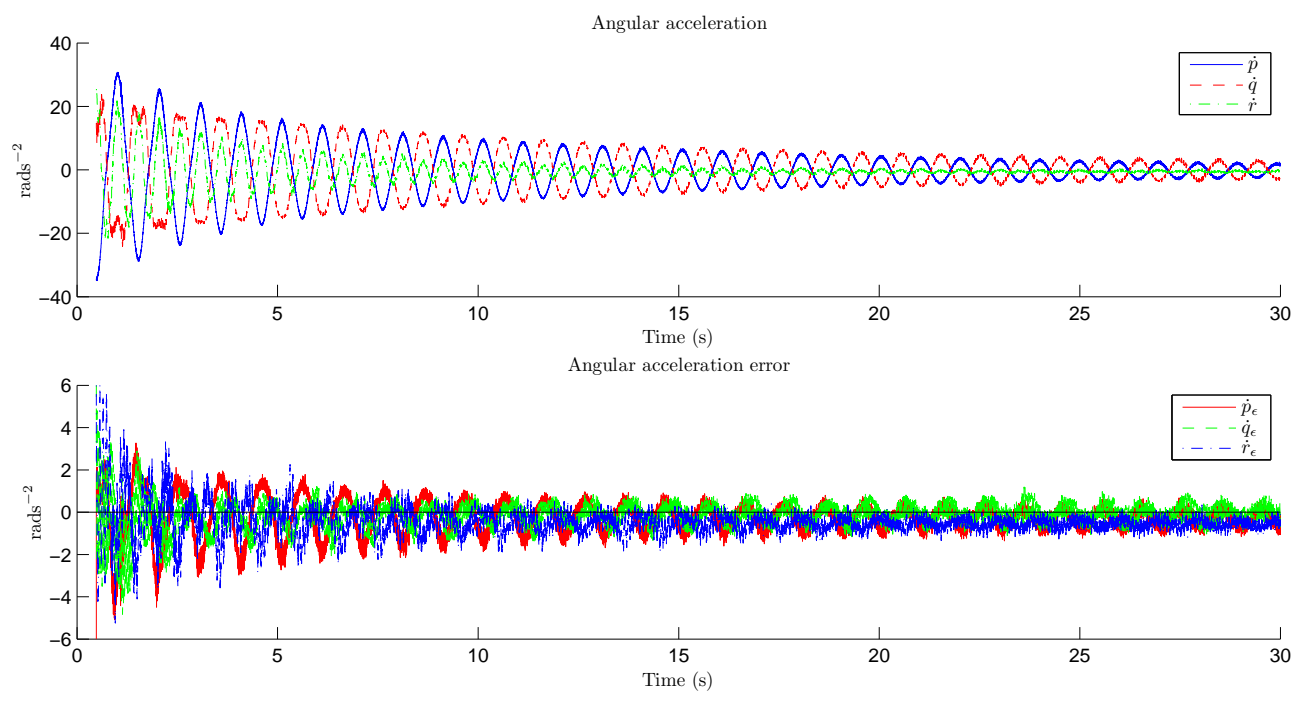

Figure 12: Angular accelerations of pendulum measured by accelerometer array (top) and residuals (bottom)

Figure 12 and Figure 13 show the pendulum kinematics states as estimated using the pseudoinverse method and data from all 10 sensors. The angular velocity estimation algorithm used a gain of $K=2$ and the orientation estimation algorithm used gains of $K_{P}=5$ and $K_{I}=1.0$. Each figure consists of 2 plots: the upper plot represents the measured or estimated state and the lower plot represents the corresponding error. Small errors in alignment calibration and the constant acceleration of gravity result in an angular velocity bias error and low velocities (see Figure 13). This error is compensated in $p$ and $q$ but cannot be compensated in $r$ as this axis of rotation eventually aligns with the direction of gravity.

To investigate the relationship between error and redundancy, the suspended body kinematics were computed using the pseudoinverse method for each combination of sensors for the available array of ten sensors, thus results from four to ten sensors were possible. Since the number of combinations for a given number of sensors is defined by $\left(\begin{array}{c}10 \\ n\end{array}\right), 210$ results were possible for 4 sensors, rising to 252 for 5 sensors and then falling to give only the single result when the fully redundant array of 10 sensors was computed. Performance is quantified as the mean of the RMS error in the $x, y$ and $z$ DoF for linear acceleration, angular acceleration and angular velocity. These results are shown as box plots in Figure 14 The results compare well with the simulation studies given in section 5. The anomalous rise apparent in the third graph of Figure 14 when all 10 sensors are used to calculate angular velocity. This is an artifact of the imperfect alignment of sensors causing the information of one sensor to dominate the calculation. This would suggest a practical system could make a confidence estimate of individual sensors and down grade sensors that might be close to saturation, becomes mechanically misaligned (or knocked), or operating at a low signal to noise ratio. 

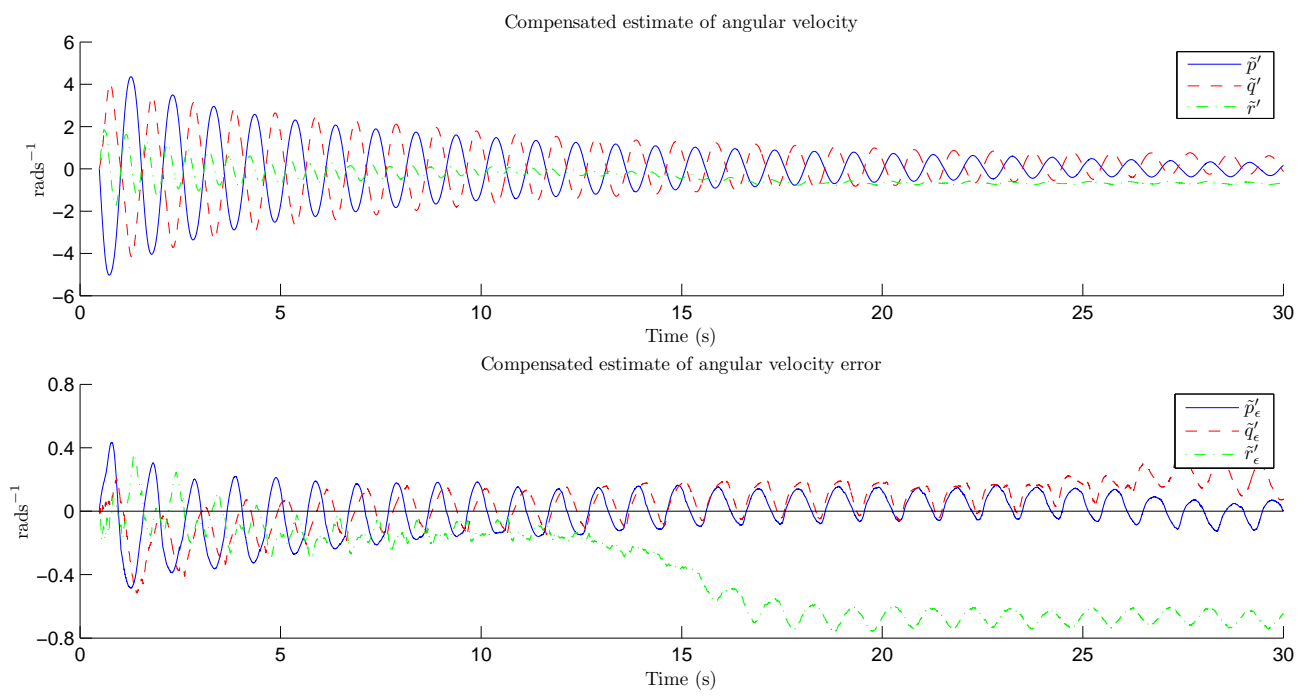

Figure 13: Estimated angular velocity compensated by orientation estimation algorithm (top) and residuals (bottom)
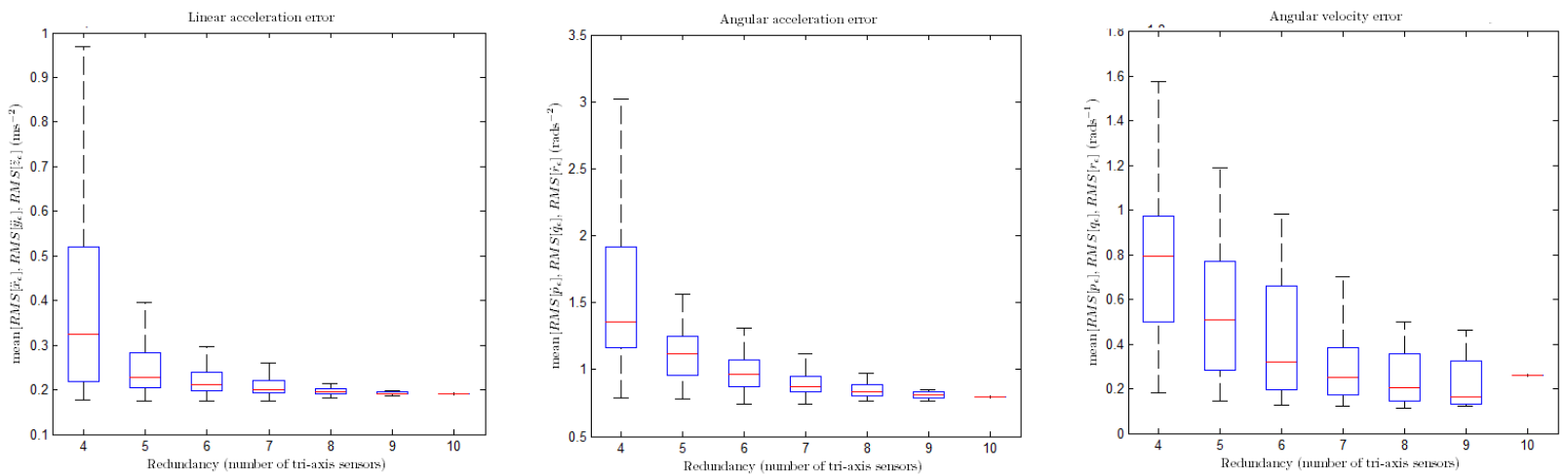

Figure 14: Relationship between error and sensor redundancy for linear acceleration (left), angular acceleration (middle), angular velocity (right)

\section{Discussion}

This paper has shown a clear advantage of higher levels of redundancy. Incorporating a large number of sensors requires a practical consideration of the computational load. The pseudoinverse method has shown to not only provide greater accuracy but to do so at significantly less computational expense. As the matrix inversion is required to be computed only once, the computational load of the pseudoinverse is proportional to the number of sensors in the array.

Due to sensor alignment errors and the constant acceleration of gravity, the estimate of angular velocity $r$ was subject to a bias error at low velocities $\left(<0.5 \mathrm{rads}^{-1}\right)$. Observations of the direction of gravity enabled the orientation estimation algorithm to compensate for such errors in $p$ and $q$; however, compensation is not possible when the axis of $r$ is parallel 
with the gravity vector. Such compensation may be achieved if the algorithm is extended to incorporate information from a magnetometer [41].

An accelerometer array cannot be used to directly determine the direction of an angular velocity. The angular velocity estimation algorithm presented can potentially assert an incorrect sign when angular velocities and accelerations are both of low magnitude. The velocity estimation method appeared to be robust in dealing with this problem although a sign switch is possible.

An accelerometer array IMU presents a number of benefits to a biomedical application. Unlike a conventional IMU it is able to provide direct measurement of angular acceleration which may provide new insight to movement studies. Redundancy provides increased levels of accuracy, potentially allowing a system to be created where a single (more expensive) high quality sensor may be substituted for many (cheaper) lower quality sensors.

Calibration has been limited to being off-line using a specific calibration dataset. If future work can achieve an on-line calibration method capable of estimating changes in individual accelerometer locations and orientations using the natural motion of the subject, then use of accelerometer arrays can be extended to non-rigid bodies. This would be of interest to biomedical applications as it would allow the use of accelerometers woven into a wearable fabric. In the interim, sensors could be embedded within a rigid orthosis such as an angle foot orthosis, or onto rigid clothing inserts. In either case, the resulting sensor will allow continuous, low power and accurate measurements with minimum obstruction to patient movements and lifestyle.

This paper has demostrated how redundancy in an array of acceleroemters can improve measurement accuracy. Redundancy of such homogenous arrays opens avenues for further exploration on object dynamics, in particular considering relaxing the constraints by considering multiple sensors attached to an elastic object, such as skin, or constrained to move in a complex relationship (such as clothing), or subject to vibration modes, (such as an aeroplane wing) or included a segmentation such as biomechanical joint was included as a constraint.

\section{Conclusion}

This paper analyses the benefits of using large arrays of accelerometers. Results include both simulation studies of up to 100 tri-axial sensors and empirical results of an array of up to 10 MEMS accelerometers. A comparison is given between simulation and empirical data based on a 2 axis pendulum simulating a knee.

Two methods are reported for the reconstruction of an acceleration state vector to represent the limb. Both methods allow the pre-computation of the necessary matrix inverse. The method based on computing a pseudoinverse (using singular value decomposition) is shown to achieve better accuracy than that based on combinatorial averaging of the sensors, and at less computational expense.

A limitation of accelerometer arrays is that it is not possible to recover the sign of the angular velocities directly so a method is given that tracks velocity transitions through this zero transition. Finally, the paper gives a method for assessing the optimal placement of sensors in a large planar array, describes a hardware solution and discusses a method for calibrating large arrays of accelerometer sensors. 


\section{Appendix A - Planar simplification}

If motion is constrained to a planar coordinate system so that translational motion occurs only in the $x$ and $y$ dimensions and rotational motion only around the $z$ axis, each sensor is reduced to a dual-axis arrangement and equations (3), (8) and (9) simplify to equations (43), (44) and (45) respectively. For planar systems the rotational velocity is denoted by $\omega$. The planar simplification gives a useful insight into the arrangement of redundant sensor constellations (see ??).

$$
\begin{gathered}
{ }^{i} \boldsymbol{\alpha}=\left[\begin{array}{ll}
\alpha_{x} & \alpha_{y}
\end{array}\right]^{T} \\
\boldsymbol{G}_{i}=\left[\begin{array}{cccc}
1 & 0 & -d_{x} & -d_{y} \\
0 & 1 & -d_{y} & d_{x}
\end{array}\right] \\
\boldsymbol{s}=\left[\begin{array}{llll}
\ddot{x} & \ddot{y} & \omega^{2} & \dot{\omega}
\end{array}\right]^{T}
\end{gathered}
$$

\section{Angular velocity estimation}

For the simplified planar system, the objective function is simply the difference between the squared estimated angular velocity term and the squared angular velocity term yielded by the state vector $\boldsymbol{s}$. The error originally defined in equation (24) in the estimated angular velocity may be redefined as the scalar quantity of equation (46).

$$
\tilde{e}=\operatorname{sign}\left(\tilde{\omega}\left(\tilde{\omega}^{2}-\omega^{2}\right)\right)|| \tilde{\omega}\left|-\sqrt{\omega^{2}}\right|
$$

\section{Appendix B - Optimal array configurations}

For ideal accelerometers, all valid array configurations perform equally. In practice, errors due to noise and signal saturation in accelerometer measurements will mean that some array configurations perform better than others. We wish to evaluate the effect of individual array characteristics to understand what determines an optimal array. An accelerometer array may be characterised by the following:

1. Position of the kinematic origin relative to a given sensor constellation;

2. Orientation of the kinematic origin relative to a given sensor constellation;

3. Sensor constellation, i.e. a set of specific sensor locations; and

4. Volume occupied by a given the sensor constellation.

Qin et al. 34] use inspection of matrix singular values to determine the suitability of a sensor configuration and determined that an optimal design is represented by an isotropic matrix; that is, a matrix with a condition number of one where all singular values are identical and nonzero. This is achieved by a sensor constellation represented by the vertices of a Platonic solid. The analysis of other authors exist for planar [45, 46] and 3D [47] [48] accelerometer arrays. 


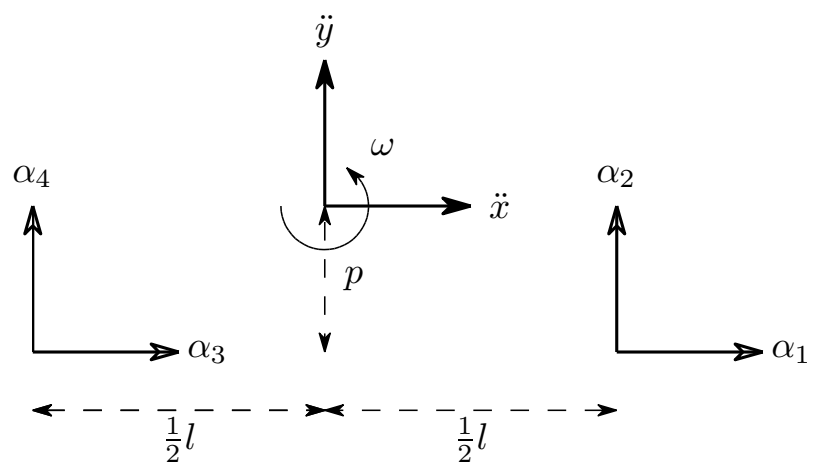

Figure 15: Schematic describing the planar sensor array characterised by parameters $p$ (origin displacement) and $l$ (sensor separation)

\section{Array volume and kinematic origin}

To analyse the effect of the kinematic origin location and array volume we will consider a simplified planar array of 2 dual-axis accelerometers represented by the measurements $\alpha_{1}^{\prime}$ to $\alpha_{4}^{\prime}$, each incorporating an error $\left(\delta_{1}\right.$ to $\left.\delta_{4}\right)$. The kinematic origin of the array is equidistant from each sensor and may be displaced by a distance $p$ along the origin $y$ axis (remaining equidistant from each sensor). The volume of the array is defined by the distance of separation, $l$. The system is described by the schematic shown in Figure 15, Equation (47) describes the forward kinematic solution.

$$
\begin{gathered}
\boldsymbol{\alpha}^{\prime}=\boldsymbol{H} \boldsymbol{s}+\boldsymbol{\delta} \\
{\left[\begin{array}{c}
\alpha_{1}^{\prime} \\
\alpha_{2}^{\prime} \\
\alpha_{3}^{\prime} \\
\alpha_{4}^{\prime}
\end{array}\right]=\left[\begin{array}{cccc}
1 & 0 & -\frac{l}{2} & p \\
0 & 1 & p & \frac{l}{2} \\
1 & 0 & \frac{l}{2} & p \\
0 & 1 & p & -\frac{l}{2}
\end{array}\right]\left[\begin{array}{c}
\ddot{x} \\
\ddot{y} \\
\omega^{2} \\
\dot{\omega}
\end{array}\right]+\left[\begin{array}{c}
\delta_{1} \\
\delta_{2} \\
\delta_{3} \\
\delta_{4}
\end{array}\right]}
\end{gathered}
$$

The inverse kinematic solution may be arranged to provide the measured kinematic states of the body origin in terms of the true kinematic states and measurement errors as shown by equations (49) and (50). In these equations, $\ddot{x}^{\prime}, \ddot{y}^{\prime}, \omega^{2^{\prime}}$ and $\dot{\omega}^{\prime}$ represent the measured kinematic states of the body origin.

$$
\begin{gathered}
\boldsymbol{s}^{\prime}=\boldsymbol{H}^{-1} \boldsymbol{\alpha}^{\prime} \\
=\boldsymbol{H}^{-1}(\boldsymbol{H} \boldsymbol{s}+\boldsymbol{\delta}) \\
=\boldsymbol{s}+\boldsymbol{H}^{-1} \boldsymbol{\delta} \\
{\left[\begin{array}{c}
\ddot{x}^{\prime} \\
\ddot{y}^{\prime} \\
\omega^{2^{\prime}} \\
\dot{\omega}^{\prime}
\end{array}\right]=\left[\begin{array}{c}
\ddot{x} \\
\ddot{y} \\
\omega^{2} \\
\dot{\omega}
\end{array}\right]+\left[\begin{array}{cccc}
\frac{1}{2} & -\frac{p}{l} & \frac{1}{2} & \frac{p}{l} \\
\frac{p}{l} & \frac{1}{2} & -\frac{p}{l} & \frac{1}{2} \\
-\frac{1}{l} & 0 & \frac{1}{l} & 0 \\
0 & +\frac{1}{l} & 0 & -\frac{1}{l}
\end{array}\right]\left[\begin{array}{l}
\delta_{1} \\
\delta_{2} \\
\delta_{3} \\
\delta_{4}
\end{array}\right]}
\end{gathered}
$$

For this simplified equidistant configuration it can be seen from equation (50) how the distance from the origin $(p)$ and sensor separation $(l)$ may be manipulated to minimise the 
error in the measured kinematics states. The error in the measured angular states $\left(\omega^{2}\right.$ and $\left.\dot{\omega}^{\prime}\right)$ is independent of the origin displacement and inversely proportional to the sensor separation. Error components in the measured linear states $\left(\ddot{x}^{\prime}, \ddot{y}^{\prime}\right)$ are proportional to the origin displacement and inversely proportional to the sensor separation. This method extends to assessing redundant planar sensor arrays, and leads to a weighted average (linear accelerations) or a weighted sum of difference (angular acceleration). Thus it can be demonstrated that an optimal array would maximise the sensor separation. The kinematics origin should be located at the point where the expected mean acceleration is minimised. Where more specific information is not available this could be considered as the centre of mass of the limb. Other authors have arbitrarily chosen the centre of volume as the kinematic origin of a sensor array [33, 34], so assuming a homogeneous body the methodology we describe gives a justification for their decision.

Analysis of the equidistant planar structure supports Qin's assertion [34] that a reasonable sensor array would be to distribute the sensors on the vertices of a Platonic solid. This only applies if the body is assumed to be rotating around the centre of the sensor constellation. Where more sensors than vertices are used in the IMU, a consideration of the singular values of the reconstruction matrix is an appropriate method to optimise sensor placement.

\section{References}

[1] Huiyu Zhou and Huosheng Hu. Human motion tracking for rehabilitation-a survey. Biomedical Signal Processing and Control, 3(1):1 - 18, 2008.

[2] D.A. Rodriguez-Silva, F. Gil-Castineira, F.J. Gonzalez-Castano, R.J. Duro, F. LopezPena, and J. Vales-Alonso. Human motion tracking and gait analysis: a brief review of current sensing systems and integration with intelligent environments. pages $166-171$, 2008.

[3] J. K. Aggarwal and Q. Cai. Human motion analysis: A review. Computer Vision and Image Understanding, 73(3):428 - 440, 1999.

[4] V.M. Pomeroy, E. Evans, and J.D. Richards. Agreement between an electrogoniometer and motion analysis system measuring angular velocity of the knee during walking after stroke. Physiotherapy, 92(3):159 - 165, 2006.

[5] Arturo Vega-Gonzlez and Malcolm H. Granat. Continuous monitoring of upper-limb activity in a free-living environment. Archives of Physical Medicine and Rehabilitation, 86(3):541 - 548, 2005.

[6] K. Aminian, B. Najafi, C. Bla, P. F. Leyvraz, and Ph. Robert. Spatio-temporal parameters of gait measured by an ambulatory system using miniature gyroscopes. Journal of Biomechanics, 35(5):689 - 699, 2002.

[7] Foo B Varma M Moran S Taub E Uswatte G, Miltner WH. Objective measurement of functional upper-extremity movement using accelerometer recordings transformed with a threshold filter. Stroke, pages 662-667, 2000. 
[8] Gitendra Uswatte, Wai Leong Foo, Harry Olmstead, Kristine Lopez, Ashleigh Holand, and Leslie Box Simms. Ambulatory monitoring of arm movement using accelerometry: An objective measure of upper-extremity rehabilitation in persons with chronic stroke. Archives of Physical Medicine and Rehabilitation, 86(7):1498 - 1501, 2005.

[9] Eva Bernmark and Christina Wiktorin. A triaxial accelerometer for measuring arm movements. Applied Ergonomics, 33(6):541 - 547, 2002.

[10] Ann V. Rowlands, Philip W. M. Thomas, Roger G. Eston, and Rodney Topping. Validation of the RT3 triaxial accelerometer for the assessment of physical activity. Medicine and Science in Sports and Exercise, 36(3):518-524, March 2004.

[11] Schutz Y. Kumahara H, Tanaka H. Daily physical activity assessment: What is the importance of upper limb movements vs whole body movements. Int $J$ Obes, pages 1105-1110, 2004.

[12] P.H. Veltink and H.M. Franken. Detection of knee unlock during stance by accelerometry. Rehabilitation Engineering, IEEE Transactions on, 4(4):395 -402, December 1996.

[13] N. Yazdi, F. Ayazi, and K. Najafi. Micromachined inertial sensors. 86(8):1640-1659, August 1998.

[14] A. Godfrey, R. Conway, D. Meagher, and G. Laighin. Direct measurement of human movement by accelerometry. Medical Engineering 83 Physics, 30(10):13641386, Dec 2008.

[15] V. Krishnan. Measurement of angular velocity and linear acceleration using linear accelerometers. Journal of the Franklin Institute, 280(4):307 - 315, 1965.

[16] Alfred R. Schuler, Anthony Grammatikos, and Kenneth A. Fegley. Measuring rotational motion with linear accelerometers. In Aerospace and Electronic Systems, IEEE Transactions on, volume AES-3, pages 465 - 472, May 1967.

[17] Kionix. Using Two Tri-Axis Accelerometers for Rotational Measurements (AN 019), Jan 2008. Application Note.

[18] Wei Dong, I-Ming Chen, K. Y. Lim, and Y. K. Goh. Measuring uniaxial joint angles with a minimal accelerometer configuration. In i-CREATe '07: Proceedings of the 1st international convention on Rehabilitation engineering E3\#38; assistive technology, pages 88-91, New York, NY, USA, 2007. ACM.

[19] Jeng-Heng Chen, Sou-Chen Lee, and Daniel B. DeBra. Gyroscope free strapdown inertial measurement unit by six linear accelerometers. JOURNAL OF GUIDANCE, CONTROL, AND DYNAMICS, 17(2):286-290, March-April 1994.

[20] Sou-Chen Lee and Cheng-Yu Liu. An innovative estimation method with own-ship estimator for an allaccelerometer-type inertial navigation system. International Journal of Systems Science, 30(12):1259-1266, 1999. 
[21] Chin-Woo Tan and Sungsu Park. Design of accelerometer-based inertial navigation systems. Instrumentation and Measurement, IEEE Transactions on, 54(6):2520-2530, 2005.

[22] Chin-Woo Tan, Kirill Mostov, and Pravin Varaiya. Feasibility of a gyroscope-free inertial navigation system for tracking rigid body motion. Research report, California Partners for Advanced Transit and Highways (PATH), Berkeley, CA, May 2000.

[23] K. Liu. Discussion on measurement of angular acceleration of a rigid body using linear accelerometers. J. Appl. Mech, 43:977978, 1976.

[24] A. J. Padgaonkar, K.W. Krieger, and A. I. King. Measurement of angular acceleration of a rigid body using linear accelerometers. J. Appl. Mech, 43:977978, 1976.

[25] D. Giansanti, V. Macellari, G. Maccioni, and A. Cappozzo. Is it feasible to reconstruct body segment 3-d position and orientation using accelerometric data? Biomedical Engineering, IEEE Transactions on, 50(4):476 -483, apr. 2003.

[26] Qi Wang, Mingli Ding, and Peng Zhao. A new scheme of non-gyro inertial measurement unit for estimating angular velocity. 2:1564 - 1567 Vol.2, 2-6 2003.

[27] Wang Xiao-xu, Xue Hong-xiang, Xia Quan-xi, and Sun Ming. Design and simulation analysis of gyroscope-free inertial measurement unit. Journal of Chinese Inertial Technology, 16(2):154-158, April 2008.

[28] Ying Kun Peng and M.F. Golnaraghi. A vector-based gyro-free inertial navigation system by integrating existing accelerometer network in a passenger vehicle. In Position Location and Navigation Symposium, 2004. PLANS 2004, pages 234-242, 26-29 2004.

[29] Jin Xudan, Mao Zheng, Wei Fuling, and Wang Yali. Research on gyroscope free strapdown inertial navigation system based on 3-axis accelerometer. pages 1-967-1-970, aug. 2007.

[30] Chi-Wei Ho and Pei chun Lin. Design and implementation of a 12-axis accelerometer suite. Intelligent Robots and Systems, 2009. IROS 2009. IEEE/RSJ International Conference on, pages $2197-2202$, oct. 2009.

[31] P. Schopp, L. Klingbeil, C. Peters, A. Buhmann, and Y. Manoli. Sensor fusion algorithm and calibration for a gyroscope-free imu. Procedia Chemistry, 1(1):1323 - 1326, 2009. ¡ce:title¿Proceedings of the Eurosensors XXIII conferencei/ce:title ¿.

[32] Patrick Schopp, Lasse Klingbeil, Christian Peters, and Yiannos Manoli. Design, geometry evaluation, and calibration of a gyroscope-free inertial measurement unit. Sensors and Actuators A: Physical, 162(2):379 - 387, 2010. ¡ce:title¿Eurosensors XXIII, 2009i/ce:title $\dot{i}$.

[33] K. Parsa, J. Angeles, and A. K. Misra. Rigid-body pose and twist estimation using an accelerometer array. Archive of Applied Mechanics, 74(3):223-236, Dec 2004. 
[34] Zhongkai Qin, Luc Baron, and Lionel Birglen. Robust design of inertial measurement units based on accelerometers. Journal of Dynamic Systems, Measurement, and Control, 131(3):031010, 2009.

[35] Paolo Cappa, Lorenzo Masia, and Fabrizio Patanè. Numerical validation of linear accelerometer systems for the measurement of head kinematics. Journal of Biomechanical Engineering, 127(6):919-928, 2005.

[36] J.P.B. Vreeburg. Analysis of the data from a distributed set of accelerometers, for reconstruction of set geometry and its rigid body motion. Technical Report NLR-TP98343, National Aerospace Laboratory NLR, 1999.

[37] K. Parsa, T.A. Lasky, and B. Ravani. Design and implementation of a mechatronic, all-accelerometer inertial measurement unit. Mechatronics, IEEE/ASME Transactions on, 12(6):640 -650, dec. 2007.

[38] Paolo Cappa, Fabrizio Patanè, and Stefano Rossi. A redundant accelerometric cluster for the measurement of translational and angular acceleration and angular velocity of the head. Journal of Medical Devices, 1(1):14-22, 2007.

[39] P. Cardou, G. Fournier, and P. Gagnon. A nonlinear program for angular-velocity estimation from centripetal-acceleration measurements. Mechatronics, IEEE/ASME Transactions on, $\mathrm{PP}(99): 1-13,2010$.

[40] Sebastian O.H. Madgwick, Andrew J.L. Harrison, and Ravi Vaidyanathan. Estimation of imu and marg orientation using a gradient descent algorithm. In International Conference on Rehabilitation Robotics (ICORR), July 2011.

[41] R. Mahony, T. Hamel, and J.-M. Pflimlin. Nonlinear complementary filters on the special orthogonal group. Automatic Control, IEEE Transactions on, 53(5):1203 -1218, june 2008.

[42] Mark Euston, Paul Coote, Robert Mahony, Jonghyuk Kim, and Tarek Hamel. A complementary filter for attitude estimation of a fixed-wing uav with a low-cost imu. In 6 th International Conference on Field and Service Robotics, July 2007.

[43] National Imagery and Mapping Agency. World geodetic system 1984: Its deffnition and relationships with local geodetic systems. Technical Report NIMA TR8350.2, U.S. Department of National Defense, January 2000.

[44] K. Parsa, J. Angeles, and A.K. Misra. Attitude calibration of an accelerometer array. Robotics and Automation, 2002. Proceedings. ICRA '02. IEEE International Conference on, 1:129 - 134 vol.1, 2002.

[45] Philippe Cardou and Jorge Angeles. Singularity analysis of accelerometer strapdowns for the estimation of the acceleration field of a planar rigid-body motion. In 12th IFToMM World Congress, 2007. 
[46] T. R. Williams and K. R. Fyfe. Planar accelerometer configurations. Journal of Applied Mechanics, 71(1):10-14, 2004.

[47] Bruno Zappa, Giovanni Legnani, Anton J. van den Bogert, and Riccardo Adamini. On the number and placement of accelerometers for angular velocity and acceleration determination. Journal of Dynamic Systems, Measurement, and Control, 123(3):552$554,2001$.

[48] G. Baselli, G. Legnani, P. Franco, F. Brognoli, A. Marras, F. Quaranta, and B. Zappa. Assessment of inertial and gravitational inputs to the vestibular system. Journal of Biomechanics, 34(6):821 - 826, 2001. 